$\mathrm{H}$ felis infected mice is likely to result in increased expressions of genes which control the influx and efflux of iron in gastrointestinal cells. Divalent metal transporter 1 (DMT1) which is negatively controlled by hepcidin is expressed on the apical membrane of gastrointestinal cells and absorbs reduced ferrous iron $\left(\mathrm{Fe}^{2+}\right)$ from the lumen. There was a significant increase $(\mathrm{p}<0.02)$ in Dmt1 gastric transcript levels in $\mathrm{H}$ felis-infected INS-GAS compared to uninfected INS-GAS mice or FVB/N controls. Other iron metabolism gene expression levels were also significantly increased with gastric $\mathrm{H}$ felis infection, such as Ferroportin 1 ( $p<0.05)$, Transferrin receptor 1 ( $p<0.003)$ and Lipocalin $2(p<0.001)$. Serum ferritin levels were significantly reduced $(p<0.001)$ in $\mathrm{H}$ felisinfected INS-GAS mice compared to uninfected controls at 9 months.

Conclusion This study demonstrates the interplay between hepcidin transcript levels and the expression of host iron metabolism genes. Previous studies have shown that chronic gastric $\mathrm{H}$ felis infection in INS-GAS mice results in decreased serum transferrin saturation. This study investigates the molecular basis for previous observations and finds that chronic gastric Helicobacter infection results in a significant reduction in gastric hepcidin gene expression levels which coincides with significant increases in gene expression of several proteins implicated in host iron metabolism.

Competing interests None.

Keywords helicobacter, hepcidin, INS-GAS mouse, iron metabolism.

\title{
PTU-096 스 HOST IRON METABOLISM GENE EXPRESSION IN INS-GAS MOUSE MODEL OF HELICOBACTER INFECTION
}

doi:10.1136/gut.2011.239301.224

M J Thomson, 1,* D M Pritchard, ${ }^{2}$ S A Boxall, ${ }^{1}$ A Varro, ${ }^{2}$ J E Crabtree ${ }^{1}$ Molecular Gastroenterology, Leeds Institute of Molecular Medicine, Leeds, UK; ${ }^{2}$ Institute of Translational Medicine, University of Liverpool, Liverpool, UK

Introduction Iron deficiency is the most common nutritional disorder globally. There is increasing evidence from clinical and population studies for a role of $\mathrm{H}$ pylori infection in the aetiology of iron deficiency. Rodent models of Helicobacter infection are needed to investigate causal links to iron deficiency in the host. The aim of this study was to investigate the effects of gastric Helicobacter infection on host iron metabolism gene expression in hypergastrinaemic INS-GAS mice.

Methods Male transgenic INS-GAS mice were inoculated with $H$ felis or broth only by oral gavage. Uninfected genetic background FVB/N mice were used as controls. Infected mice and uninfected controls were sacrificed at 9 months post-inoculation. Gastric corpus mucosa was stored for total RNA extraction and analysis by quantitative real time PCR. The level of expression of target genes was assessed as relative abundance compared to Gapdh control gene by the $\Delta \Delta$ Ct method. Serum ferritin protein levels were measured by ELISA.

Results H felis-infected INS-GAS mice had a significant reduction in hepcidin transcript levels in the gastric mucosa compared to uninfected INS-GAS mice and FVB/N controls $(p<0.03)$. The reduction in hepcidin hormone expression in 